\title{
Small-sized type A thymoma with pulmonary metastasis: a case report
}

\author{
Sachi Kawagishi ${ }^{\text {* }}$, Tomohiro Maniwa ${ }^{1}$, Hirokazu Watari ${ }^{1}$, Ryuhei Sakata ${ }^{1}$, Akiisa Omura' ${ }^{1}$ Ryo Tanaka', \\ Toru Kimura ${ }^{1}$, Keiichiro Honma ${ }^{2}$ and Jiro Okami ${ }^{1}$
}

\begin{abstract}
Background: Type A thymomas comprise a homogenous population of neoplastic epithelial cells that are characterized by a spindle/oval shape without nuclear atypia. They may be accompanied by few non-neoplastic lymphocytes. Most type A thymomas are detected in the earlier Masaoka stages. Compared to other thymoma subtypes, they rarely metastasize or recur. There have been some reports of patients with type A thymomas with pulmonary metastasis; however, these thymomas were $20 \mathrm{~mm}$ or more in size. Herein, we report the case of a patient who underwent surgical resection for a small-sized type A thymoma $(12 \mathrm{~mm})$ with pulmonary metastasis.

Case presentation: A 62-year-old patient presented with an abnormal shadow in the left lung on plain chest radiography during a medical checkup. Chest computed tomography revealed a 12-mm tumor in the anterior mediastinum and a 13 -mm nodule in the left lower lobe. ${ }^{18} \mathrm{~F}$-fluorodeoxyglucose positron emission tomography/computed tomography revealed uptake in the anterior mediastinal tumor, but did not show a significant uptake in the pulmonary nodule. The patient underwent surgical resection on two separate occasions, and was diagnosed with an atypical type A thymoma and pulmonary metastasis. The TNM classification was p-T1aNOM1 b stage IVb, and it was stage IVb according to the Masaoka staging system. No recurrence was observed during the follow-up.
\end{abstract}

Conclusions: We report a case of the smallest type A thymoma with pulmonary metastasis. Pulmonary metastasis secondary to a type A thymoma should be considered even if the thymoma is small in size ( $<20 \mathrm{~mm})$.

Keywords: Atypical type A thymoma, Thymus, Pulmonary metastasis, Anterior mediastinal tumor

\section{Background}

Type A thymomas comprise a homogenous population of neoplastic epithelial cells that are characterized by a spindle/oval shape without nuclear atypia. They may be accompanied by a few non-neoplastic lymphocytes. Type A thymomas are associated with a favorable clinical course. Compared to other thymoma subtypes, they rarely metastasize or recur $[1,2]$. However, some authors have reported cases of type A thymomas with pulmonary metastasis, wherein the thymomas were $20 \mathrm{~mm}$ or more in size [3-8]. We report the case of a patient who

\footnotetext{
*Correspondence: sachi.kawagishi@oici.jp

${ }^{1}$ Department of General Thoracic Surgery, Osaka International Cancer

Institute, 1,3,4: 3-1-69, Otemae, Chuo-ku, Osaka 541-8567, Japan

Full list of author information is available at the end of the article
}

underwent surgical resection for a small-sized $(12 \mathrm{~mm})$ atypical type A thymoma and pulmonary metastasis.

\section{Case presentation}

A 62-year-old man presented with an abnormal shadow in the left lung on plain chest radiography during a medical checkup. Chest computed tomography (CT) revealed a nodule in the left lower lobe and a tumor with calcification in the anterior mediastinum. The well-defined hilar nodule of the left lung measured $13 \mathrm{~mm}$ and was located in $\mathrm{S}^{10}$. The anterior mediastinal tumor measured $12 \mathrm{~mm}$ in size, and there was no evidence of infiltration into the adjacent tissue (Fig. 1a, b). While ${ }^{18} \mathrm{~F}$-fluorodeoxyglucose positron emission tomography/CT (FDG-PET/ $\mathrm{CT}$ ) revealed uptake in the anterior mediastinal tumor $\left(\mathrm{SUV}_{\max }=1.5\right)$, it did not reveal significant uptake in the 


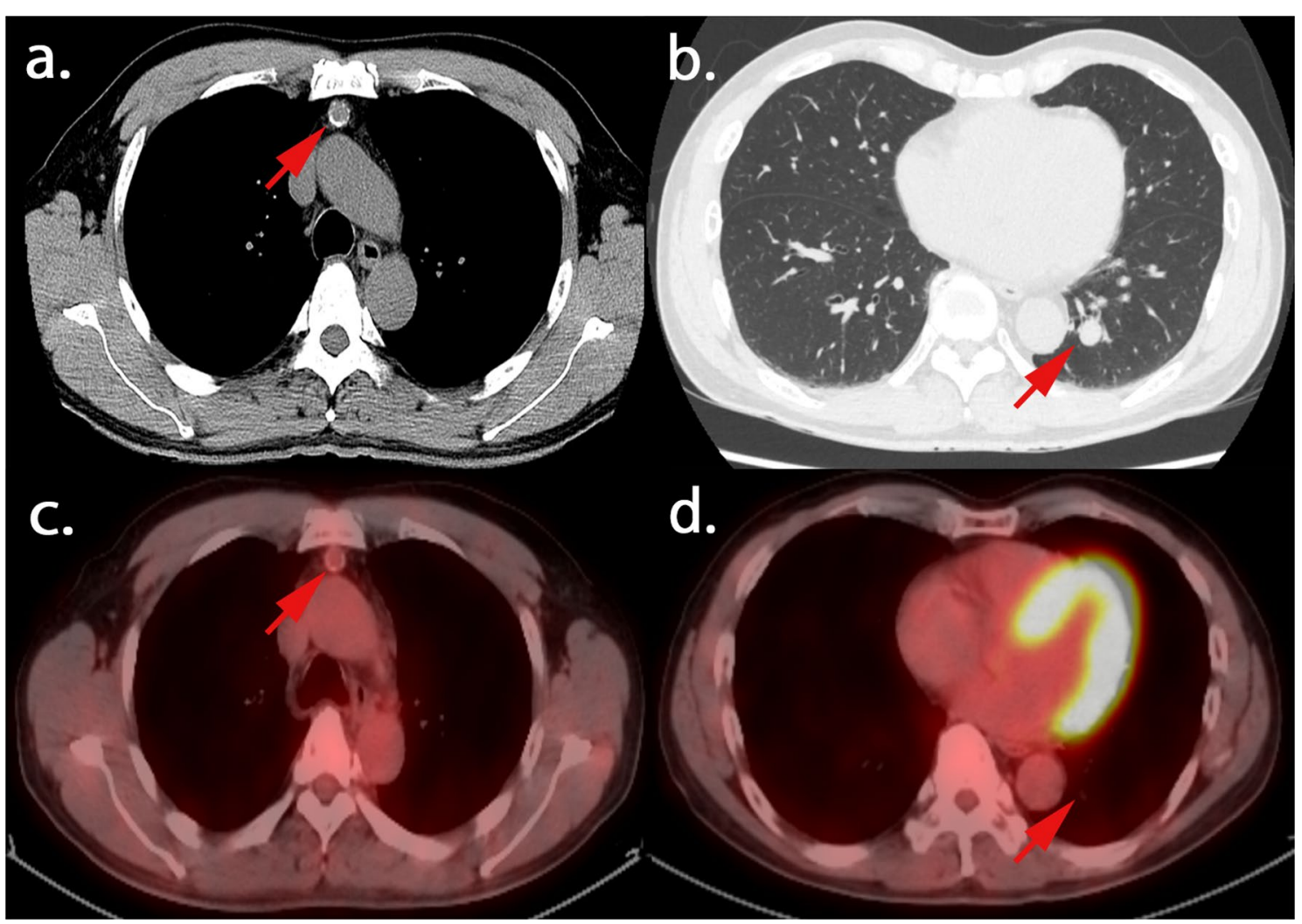

Fig. $1 \mathbf{a}, \mathbf{b}$ Chest computed tomography findings. A tumor with calcification is located in the anterior mediastinum (a) and a well-defined hilar nodule is located in $\mathrm{S}^{10}$ of the left lung $(\mathbf{b}) . \mathbf{c}, \mathbf{d}^{18} \mathrm{~F}$-fluorodexyglycose positron emission tomography/computed tomography findings. Uptake is noted in the anterior mediastinal tumor $\left(S_{U V} V_{\max }=1.5\right)(\mathbf{c})$, but no significant uptake is noted in the pulmonary nodule $\left(S U V_{\max }=1.1\right)(\mathbf{d})$

left lower-lobe nodule $\left(\mathrm{SUV}_{\max }=1.1\right)$ (Fig. 1c, d). The patient was seronegative for anti-acetylcholine receptorbinding antibodies and tumor markers. The bronchoscopy was negative for pulmonary nodule, and FDG-PET/ CT did not reveal significant uptake. From these findings, the pulmonary nodule was suggested to be a benign tumor. Surgical resection for diagnostic treatment was planned for the anterior mediastinal tumor, and pulmonary resection was planned for later. Contrast-enhanced chest $\mathrm{CT}$ revealed that the anterior mediastinal tumor was adjacent to the left brachiocephalic artery, and the border between them was unclear; therefore, the possibility of the adhesions between the tumor and artery was considered. Thymectomy was performed via a median sternotomy in consideration of safety. The operation time was $134 \mathrm{~min}$ and the bleeding volume was $75 \mathrm{~mL}$. The tumor measured $16 \mathrm{~mm} \times 13 \mathrm{~mm} \times 9 \mathrm{~mm}$. The postoperative course was uneventful, and the patient was discharged 7 days after the operation. The pathological diagnosis was of an atypical type A thymoma. Pathological examination revealed that the tumor cells exhibited poor atypia, had round nuclei, and grew in a fascicular pattern. The tumor had a hemangiopericytoma-like vascular pattern. It also had hypercellularity, but no increased mitotic counts and focal necrosis were observed. The tumor was almost encapsulated by the fibrous cap. Microscopic invasion into the surrounding adipose fatty tissue beyond the capsular portion was noted in one part of the tumor (Fig. 2).

A pulmonary nodule resection was planned after the surgery; however, the patient refused to undergo it because of his poor physical condition. Fourteen months after the surgery, follow-up chest $\mathrm{CT}$ revealed that the left lower-lobe nodule had enlarged to $18 \mathrm{~mm}$ (Fig. 3a, b). FDG-PET/CT revealed a slight uptake $\left(\mathrm{SUV}_{\max }=1.3\right)$ in the nodule. The nodule itself was considered a low-grade malignant tumor (Fig. 3c). Surgical resection for diagnostic treatment was planned to rule out malignancy. Sixteen months after the initial surgery for the thymoma, the patient underwent left lower lobe lobectomy and lymph node dissection. The nodule of the left lower lobe was near the segmental bronchus (Fig. 3b). We considered that we could not take a sufficient resection margin of the tumor if we performed sublobar resection. The operation time was $190 \mathrm{~min}$, and the bleeding volume was $20 \mathrm{~mL}$. The tumor measured $13 \mathrm{~mm} \times 12 \mathrm{~mm} \times 8 \mathrm{~mm}$. 


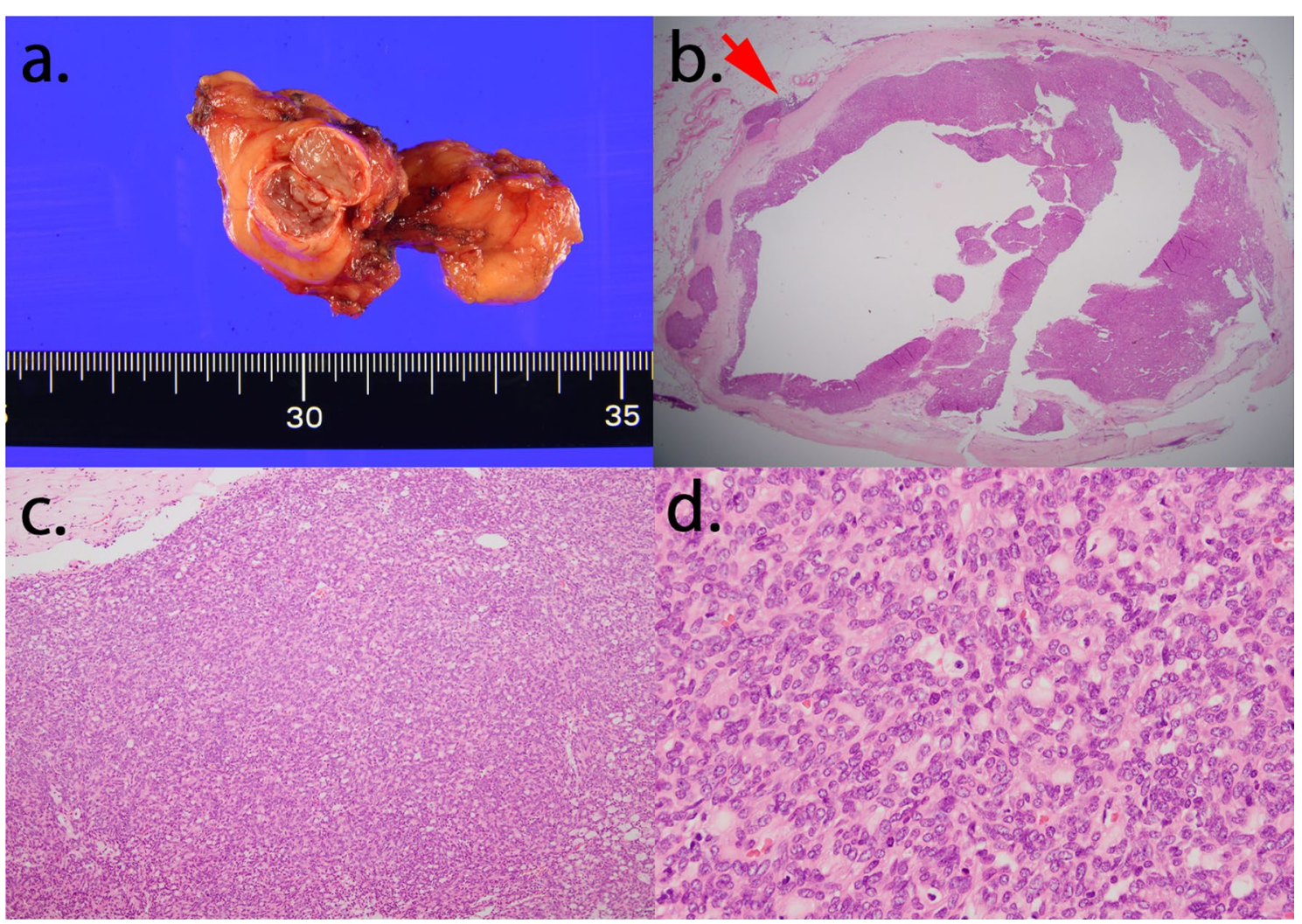

Fig. 2 a Macroscopic image of the excised thymic tumor. b-d Hematoxylin and eosin staining of the anterior mediastinal tumor. Microscopic invasion into the surrounding adipose fatty tissue beyond the capsular portion is noted only in one part of the tumor (b, arrow). Tumor cells with relatively poor atypia and round nuclei are seen to grow in a fascicular pattern. The tumor shows findings of hypercellularity (c, d)

The postoperative course was uneventful, and the patient was discharged 8 days after the operation. Postoperative pathological findings revealed densely scattered spindle cells. Immunohistochemical analysis revealed that the tumor cells were positive for the anti-pan cytokeratin antibody, p40, and paired box protein 8 . The tumor exhibited hypercellularity. However, the mitotic count was not increased, and focal necrosis was not observed (Fig. 4). The tumor was diagnosed as a metastasis of the atypical type A thymoma. The TNM classification was p-T1aNOM1b stage IVb, and it was stage IVb according to the Masaoka staging system. The tumor was completely resected, and the patient did not receive adjuvant therapy. The patient experienced no recurrence for 21 months after the surgery for thymoma and for 5 months after the pulmonary resection.

\section{Discussion}

Thymomas originate from the epithelial cells of the thymus. These tumors are unique due to their ability to partially promote T-lymphocyte maturation. Moreover, no anaplastic cells were noted in the present case. In case of type A thymomas, the occurrence of metastases and tumor-related death are generally related to high-stage disease (Masaoka stages III and IV) and incomplete tumor resection $[1,9]$. In the previously reported cases of type A thymomas, detection was during the earlier Masaoka stages (Masaoka stages I and II) and the tumors rarely metastasized [1]. The atypical type A thymoma variant was added to the World Health Organization classification as a new subtype in 2015. It may be associated with postoperative recurrence or distant metastases [10].

Previous reports have described pulmonary metastases of type A thymomas along with the thymoma sizes (Table 1) [3-8]. In these cases, there were almost no differences between the typical and atypical type A thymomas. Despite thymoma invasion, these patients had pulmonary metastasis. In previous cases of type A thymomas with pulmonary metastasis, the primary tumors were larger than $20 \mathrm{~mm}$. However, in the present case, the primary tumor measured $12 \mathrm{~mm}$ in size, and the metastatic tumor was larger than the thymoma.

This report presents a case of the smallest type A thymoma with pulmonary metastasis reported yet. Due to the smaller size of the thymoma, the pulmonary nodule 


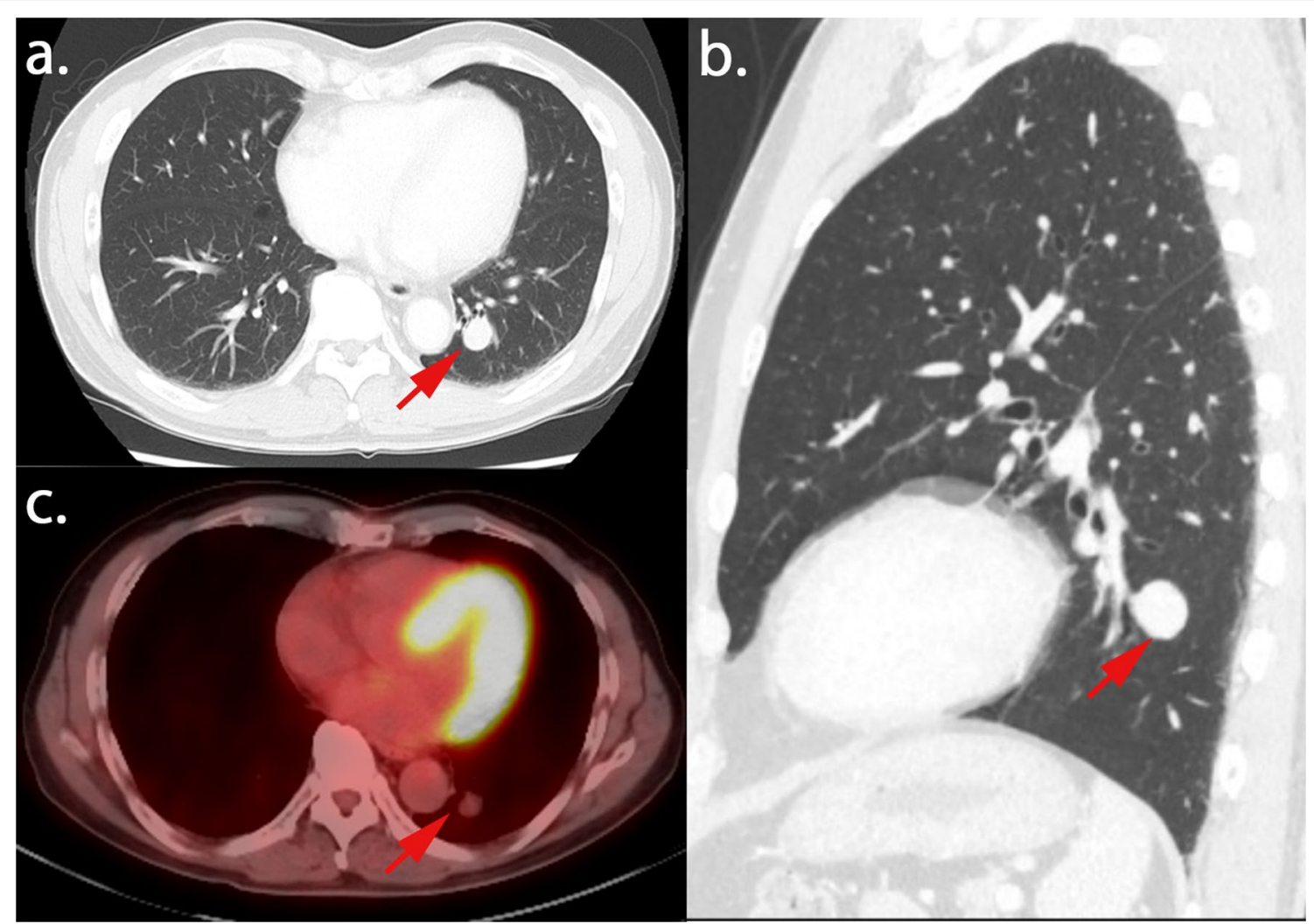

Fig. $3 \mathbf{a}$, b Chest computed tomography findings. The nodule of the left lower lobe increased in size, and it was near the segmental bronchus. $\mathbf{c}$ ${ }^{18}$ F-fluorodexyglycose positron emission tomography/computed tomography reveal a little uptake $\left(S U V_{\max }=1.3\right)$

Table 1 Previous reports that describe a type A thymoma with pulmonary metastasis and thymoma size

\begin{tabular}{|c|c|c|c|c|c|c|c|c|}
\hline Case no. & Author & Year & Age (years) & Sex & $\begin{array}{l}\text { Pathology of } \\
\text { the thymoma }\end{array}$ & $\begin{array}{l}\text { Size of the } \\
\text { thymoma } \\
(\mathrm{mm})\end{array}$ & $\begin{array}{l}\text { Size of the } \\
\text { pulmonary } \\
\text { metastasis }(\mathrm{mm})\end{array}$ & Invasion of the thymoma \\
\hline 1 & Hirono et al. [3] & 2014 & 74 & M & - & 30 & 20 & None \\
\hline 2 & Hashimoto et al. [4] & 2017 & 35 & $\mathrm{~F}$ & Atypical & 60 & - & None \\
\hline 3 & Burger et al. [5] & 2017 & 72 & M & Typical & 70 & - & Mediastinal fat \\
\hline 4 & & & 64 & M & Typical & 28 & 20 & - \\
\hline 5 & & & 66 & M & Atypical & 70 & - & - \\
\hline 6 & Mengoli et al. [6] & 2017 & 55 & M & - & 44 & 8 & Fibrous capsule \\
\hline 7 & Kawakita et al. [7] & 2018 & 84 & M & Atypical & 44 & $2-5$ & Vascular \\
\hline 8 & Tatematsu et al. [8] & 2021 & 79 & $\mathrm{~F}$ & Typical & 21 & 3 & - \\
\hline 9 & Our case & 2021 & 62 & M & Atypical & 12 & 13 & Fibrous capsule \\
\hline
\end{tabular}

$M$ male, $F$ female

was not identified as a metastatic lesion. In cases wherein the type A thymoma measures less than $20 \mathrm{~mm}$, pulmonary metastasis should still be considered. Complete surgical resection is the most important prognostic factor in thymomas with local infiltration or metastasis [11].
Therefore, aggressive resection is warranted in cases wherein the thymoma and pulmonary metastasis can be resected completely. Considering the possibility of recurrence, a long-term follow-up is necessary. 


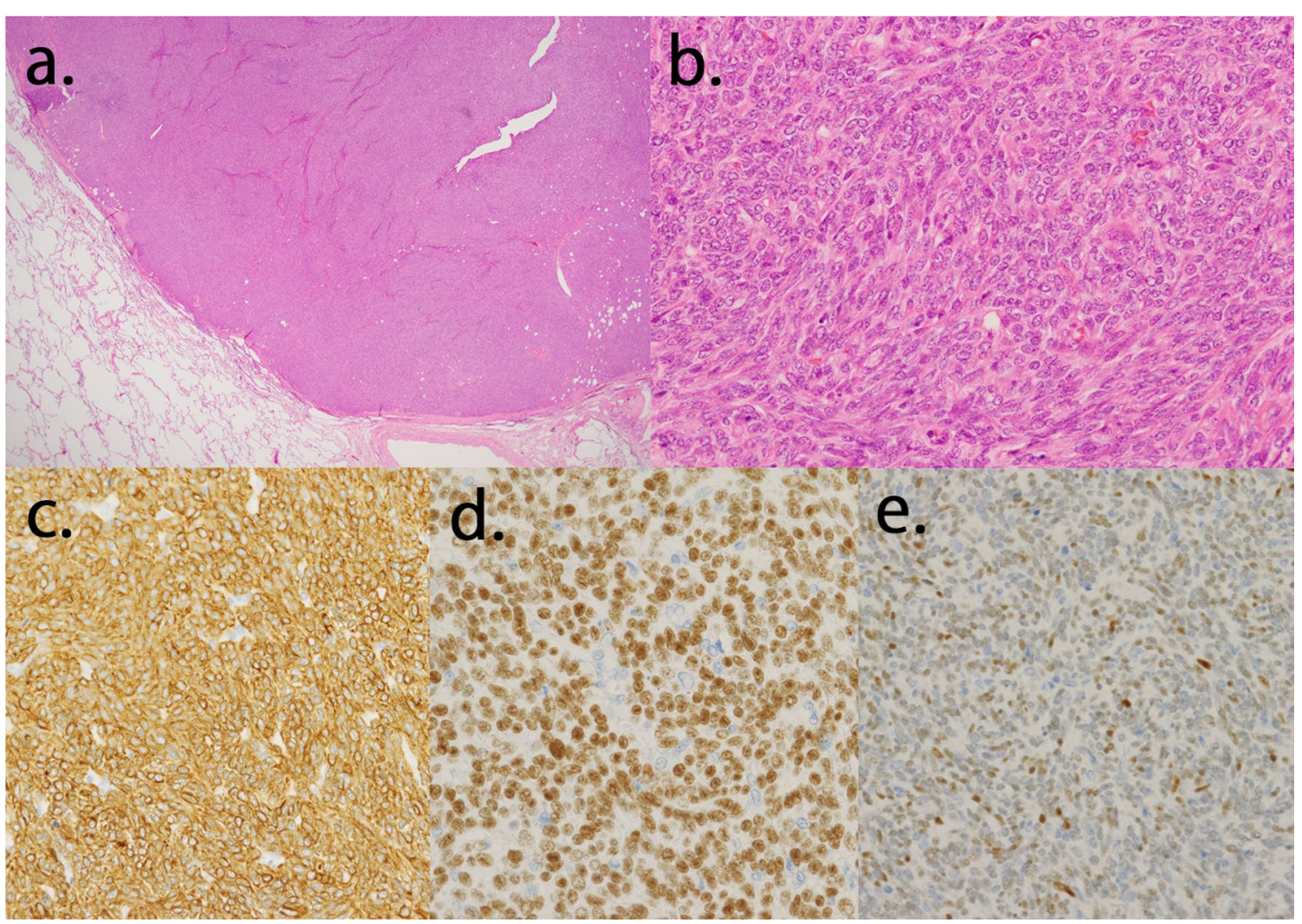

Fig. 4 a, $\mathbf{b}$ Hematoxylin and eosin staining of the pulmonary nodule. Pathological images are similar to those of an anterior mediastinal tumor. c-e An immunohistochemical analysis of the pulmonary disease. The tumor is noted to be positive for the anti-pan cytokeratin antibody (c), p40 (d), and paired box protein 8 (e)

\section{Conclusions}

We report the case of a patient who underwent surgical resection for an atypical type A thymoma and solitary pulmonary metastasis. In cases of a type A thymoma with a pulmonary nodule, pulmonary metastasis secondary to the thymoma should be considered even when the type A thymoma measures less than $20 \mathrm{~mm}$, as in our case.

\section{Abbreviations}

CT: Computed tomography; FDG-PET: ${ }^{18} \mathrm{~F}$-fluorodeoxyglucose positron emission tomography.

\section{Acknowledgements}

We thank Dr. Keiichiro Honma for the pathologic diagnosis.

\section{Authors' contributions}

SK conceptualized and designed the article and is the corresponding author. TM and JO supervised the editing of the manuscript. KH performed the histopathological diagnosis. Other remaining co-authors collected the data and discussed the content of the manuscript. All authors read and approved the final manuscript.

\section{Funding}

This study was not funded.

\section{Availability of data and materials}

The patient data of this case report will not be shared to ensure patient confidentiality.

\section{Declarations}

\section{Ethics approval and consent to participate}

The study protocol was approved by the Ethical Review Board for Clinical Studies at the Osaka International Cancer Institute.

\section{Consent for publication}

Informed consent was obtained from the patient and the patient's family for the publication of this case report.

\section{Competing interests}

The authors declare no conflicts of interest in association with the present study.

\section{Author details}

${ }^{1}$ Department of General Thoracic Surgery, Osaka International Cancer Institute, 1,3,4: 3-1-69, Otemae, Chuo-ku, Osaka 541-8567, Japan. ${ }^{2}$ Department of Pathology, Osaka International Cancer Institute, 1,3,4: 3-1-69, Otemae, Chuo-ku, Osaka 541-8567, Japan.

Received: 7 November 2021 Accepted: 11 January 2022

Published online: 20 January 2022 


\section{References}

1. Okumura M, Ohta M, Tateyama H, Nakagawa K, Matsumura A, Maeda $\mathrm{H}$, et al. The World Health Organization histologic classification system reflects the oncologic behavior of thymoma: a clinical study of 273 patients. Cancer. 2002:94:624-32.

2. Ruffini E, Filosso PL, Mossetti C, Bruna MC, Novero D, Lista P, et al. Thymoma: inter-relationships among World Health Organization histology, Masaoka staging and myasthenia gravis and their independent prognostic significance: a single-centre experience. Eur J Cardiothorac Surg. 2011:40:146-53.

3. Hirono M, Nonaka M, Himuro N, Tomita Y, Kataoka D, Kadokura M. Two cases of thymoma with pulmonary metastasis: a case report. World J Surg Oncol. 2014;12:114

4. Hashimoto M, Tsukamoto Y, Matsuo S, Nakamichi T, Kondo N, Hasegawa S. Lung metastases in an atypical type A thymoma variant. J Thorac Dis. 2017:9:E805-7.

5. Bürger T, Schaefer IM, Küffer S, Bohnenberger H, Reuter-Jessen K, Chan JK, et al. Metastatic type A thymoma: morphological and genetic correlation. Histopathology. 2017;70:704-10.

6. Mengoli MC, Longo L, Varini S, Rossi G, Lococo F. Invasive medullary type A thymoma with recurrent distant metastases. Ann Thorac Surg. 2017; 103:e423-5

7. Kawakita N, Kondo K, Toba H, Yoneda A, Takizawa H, Tangoku A. A case of atypical type $A$ thymoma with vascular invasion and lung metastasis. Gen Thorac Cardiovasc Surg. 2018;66:239-42.

8. Tatematsu T, Okuda K, Endo K, Hattori H, Matsui T, Oda R, et al. Type A thymoma with simultaneous solitary intrapulmonary metastasis: a case report. Thorac Cancer. 2021;12:1923-6.

9. Nonaka $\mathrm{D}$, Rosai J. Is there a spectrum of cytologic atypia in type a thymomas analogous to that seen in type B thymomas? A pilot study of 13 cases. Am J Surg Pathol. 2012:36:889-94.

10. Marx A, Chan JK, Coindre JM, Detterbeck F, Girard N, Harris NL, et al. The 2015 World Health Organization classification of tumors of the thymus: continuity and changes. J Thorac Oncol. 2015;10:1383-95.

11. Kondo K, Monden Y. Therapy for thymic epithelial tumors: a clinical study of 1,320 patients from Japan. Ann Thorac Surg. 2003;76:878-84

\section{Publisher's Note}

Springer Nature remains neutral with regard to jurisdictional claims in published maps and institutional affiliations.

\section{Submit your manuscript to a SpringerOpen ${ }^{\odot}$ journal and benefit from:}

- Convenient online submission

- Rigorous peer review

- Open access: articles freely available online

- High visibility within the field

- Retaining the copyright to your article 